\title{
The parental origin correlates with the karyotype of human embryos developing from tripronuclear
} zygotes

\author{
Mette Warming Joergensen ${ }^{1,2}$, Rodrigo Labouriau ${ }^{3}$, Johnny Hindkjaer ${ }^{4}$, Magnus Stougaard ${ }^{2}$, Steen Kolevraa ${ }^{1}$, Lars Bolund $^{5}$, \\ Inge Errebo Agerholm ${ }^{6}$, Lone Sunde ${ }^{5,7}$ \\ 'Department of Clinical Genetics, Vejle Hospital, Vejle; ${ }^{2}$ Department of Pathology, Aarhus University Hospital, Aarhus; ${ }^{3}$ Department of Mathematics, \\ Aarhus University, Aarhus; ${ }^{4}$ The Fertility Clinic and Centre for Preimplantation Genetic diagnosis, Aarhus University Hospital, Skejby; ${ }^{5}$ Department of \\ Biomedicine, Aarhus University, Aarhus; ${ }^{6}$ The Fertility Clinic, Horsens Hospital, Horsens; ${ }^{7}$ Department of Clinical Genetics, Aarhus University Hospital, \\ Skejby, Denmark
}

Objective: It has previously been suggested that embryos developing from intracytoplasmic sperm-injected (ICSI) zygotes with three pronuclei (3PN) are endowed with a mechanism for self-correction of triploidy to diploidy. 3PN are also observed in zygotes after conventional in vitro fertilization (IVF). The parental origin, however, differs between the two fertilization methods. Whereas the vast majority of 3PN IVF zygotes are of dispermic origin and thus more likely to have two centrioles, the 3PN ICSI zygotes are digynic in origin and therefore, more likely to have one centriole. In the present study, we examine whether the parental origin of 3PN embryos correlates with the karyotype.

Methods: The karyotype of each nucleus was estimated using four sequential fluorescence in situ hybridizations - each with two probes-resulting in quantitative information of 8 different chromosomes. The karyotypes were then compared and correlated to the parental origin.

Results: 3PN ICSI embryos displayed a significantly larger and more coordinated reduction from the assumed initial 3 sets of chromosomes than 3PN IVF embryos.

Conclusion: The differences in the parental origin—and hence the number of centrioles—between the 3PN IVF and the 3PN ICSI zygotes are likely to be the cause of the differences in karyotypes.

Keywords: Centrioles; Embryos; Human; Ploidy; Preimplantation; Tripronuclear

\section{Introduction}

Triploidy is one of the most common chromosome abnormalities in

Received: Dec 18, 2014 · Revised: Dec 29, 2014 · Accepted: Jan 19, 2015 Corresponding author: Mette Warming Joergensen

Department of Pathology, Aarhus University Hospital, Tage Hansens Gade 2, $5 \mathrm{G}$ 8000 Aarhus C, Denmark

Tel: +4561692244 Fax:+4578467499 E-mail:mejoer@rm.dk.

*The study was supported by Vejle Hospital, Vejle, University of Southern Denmark, the Aase and Einar Danielsen Foundation, the Augustinus Foundation, the Medical Research Foundation Central Region Denmark, and A.P. Moeller and Wife Chastine Mc-Kinney Moellers Foundation.

This is an Open Access article distributed under the terms of the Creative Commons Attribution Non-Commercial License (http://creativecommons.org/licenses/by-nc/3.0/) which permits unrestricted non-commercial use, distribution, and reproduction in any medium, provided the original work is properly cited. human gestation, as it is present in $1 \%-2 \%$ of pregnancies [1]. However, triploid pregnancies rarely come to term, and the very diverse phenotypes seen, are all grossly abnormal [1,2]. Triploidy may originate from either digynic or diandric fertilizations, involving either a diploid oocyte or dispermic fertilization of an oocyte [3-5].

Triploidy is also found in vitro and occurs in 4\%-7\% of in vitro fertilized $[4,6,7]$ and $6 \%$ of intracytoplasmic sperm-injected (ICSI) zygotes [4]. The vast majority of triploid embryos are recognized before transfer to the woman, by the appearance of 3 pronuclei (3PN) in the zygote instead of the normal 2. Otherwise, 3PN embryos and embryos with two pronuclei (2PN) have the same morphological appearance [8]. Analyses of 3PN zygotes after in vitro fertilization (IVF) have indicated that at least $86 \%$ originate from dispermic fertilization of the 
oocyte [9]. Since only one spermatozoon is injected into the oocyte at ICSI fertilization, the extra pronucleus in 3PN ICSI embryos is most likely of maternal origin. As the centriole is paternally inheritedsupplied as an organelle by the sperm-3PN IVF and ICSI zygotes tend to have different numbers of centrioles $[10,11]$.

Although it has been demonstrated that 3PN zygotes have 3 haploid sets of chromosomes [3,10,12-15], several studies of cleavage stage embryos arising from 3PN zygotes have revealed karyotypes that are triploid, diploid, or severely abnormal including mosaicism $[4,6,8,12,13,16-18]$. Conclusions from these studies are, however, scarce as only a few nuclei from each embryo have been analyzed, or a low number of chromosomes have been analyzed from each nucleus $[4,6,8,12,13,16-18]$.

Only a few studies have analyzed any potential differences in karyotypes between 3PN IVF and 3PN ICSI embryos [4,6,12,18], and only two studies stratified cases according to the embryonic age at which the embryos were analyzed [19,20].

Therefore, further analyses of the karyotypes in 3PN IVF and 3PN ICSI embryos could provide essential insight into early embryo development, the role of centrioles in human embryogenesis, and basic genetics.

In the present study, 163 nuclei collected from 37 3PN embryos were analyzed for ploidy using fluorescence in situ hybridization (FISH) probes for 8 different chromosomes. The results were analyzed with a focus on the fertilization method (IVF or ICSI) and the embryonic age.

\section{Methods}

\section{Ethical approval}

Between May 1, 2010, and June 30, 2011, all patients undergoing treatment at the Fertility Clinic of Braedstrup/Horsens Hospital were asked for permission to include their 3PN zygotes in the study.

Oral and written informed consent was obtained from all participants before inclusion. The Regional Committees on Biomedical Research Ethics in Southern Denmark and the Danish Data Protection Agency approved the study.

\section{Participants and IVF}

Patients underwent treatment according to the guidelines in the clinic; that is, standard IVF was performed in all cases unless there were (1) less than 1 million spermatozoa/mL after preparation, (2) low spermatozoa motility, or (3) a history of IVF failure. In these instances, standard ICSI procedures were followed.

\section{Embryo culture}

Immediately after sperm addition or injection, fertilized embryos were cultured under oil at $37^{\circ} \mathrm{C}, 6 \% \mathrm{CO}$, and $5 \% \mathrm{O}_{2}$ in a sequential culture medium (Cook Sydney IVF Fertilization/Cleavage/Blastocyst Medium, Cook, Sydney, Australia) changed on days 3 and 5. At 18-20 hours after injection or addition of sperm, the oocytes were checked for fertilization via the counting of pronuclei. If a 3PN zygote was observed and the patient had consented, the zygote was included in the study.

\section{Embryo fixation}

Artificial arrest of the developing 3PN embryos was performed at different points in time between day 0 and day 5. During the fixation procedure, embryos were observed using an inverted microscope.

Embryos were removed from the culture medium and incubated in a drop of Tyrode's acid in order to remove the zona pellucida. Just before complete disintegration of the zona pellucida, the embryos were transferred to a drop of phosphate buffered saline (PBS). The embryos were then transferred to a poly-L-lysine slide (Menzel-Glaser, Germany) along with $0.01-\mathrm{mol} / \mathrm{L}$ hydrogen chloride $(\mathrm{HCl})$ in $0.1 \%$ polysorbate 20 (Tween 20). At this point, the cell membrane and the cytoplasm began to disintegrate, leaving the nuclei clearly visible and fixed (after a few seconds to several minutes). The location of the nuclei was registered and marked by a diamond pen. Slides were airdried followed by washing in PBS and dehydrated using an ascending ethanol series. The slides were stored at $-80^{\circ} \mathrm{C}$ until the hybridization protocol was performed. For each embryo, the number of cells observed before fixation and the number of nuclei observed after fixation were noted.

\section{FISH}

FISH of the fixed nuclei was performed using four sequential hybridizations. Centromeric probes for chromosomes 6 and 11 (first hybridization), 8 and 17 (second hybridization), 10 and 18 (third hybridization), and $X$ and $Y$ (fourth hybridization) were used-all commercially available from Cytocell, Cambridge, United Kingdom.

The hybridization protocol, in brief, was as follows: Slides were incubated with pepsin $(100 \mu \mathrm{g} / \mathrm{mL})$ for 10 minutes at $37^{\circ} \mathrm{C}$, rinsed in bidistilled water and PBS, and fixed for 5 minutes in 1\% paraformaldehyde in PBS at room temperature. The slides were then rinsed in PBS and bi-distilled water and dehydrated using an ascending ethanol series. The probes were added to the slide under a coverslip. The probe and the nuclear DNA were denatured at $75^{\circ} \mathrm{C}$ for 3 minutes and left to hybridize in a humidity chamber at $37^{\circ} \mathrm{C}$ for 1 hour. To remove an unhybridized probe, the slides were washed in 50\% formamide in saline-sodium citrate buffer (SSC) at $42^{\circ} \mathrm{C}$ for 5 minutes, followed by washing in SSC and $0.05 \%$ polysorbate 20 (Tween 20) at room temperature for 5 minutes. The slides were dehydrated and mounted in Vectashield (Vector Laboratories, USA) antifade medium 
containing $1 \mathrm{ng} / \mathrm{mL}$ 4,6-diamidine-2-phenylindole to counterstain the nuclei and examined.

In order to perform rehybridization, the slides were washed twice in SSC and $0.05 \%$ polysorbate 20 (Tween 20) at room temperature for 5 minutes, followed by 2 minutes of washing in PBS and then dehydrated through an ascending ethanol series, after which the denaturation and hybridization could proceed as mentioned above.

The nuclei were analyzed using an epifluorescence microscope (Leica DM6000B) and the images of every nucleus were recorded vertically ( $z$-stacking) with an average distance between images of $0.8 \mu \mathrm{m}$ using a digital monochrome CCD camera (DFC360FX) operated by the Leica Application Suite Advanced Fluorescence software.

A microscope slide containing lymphocyte nuclei from a male with a normal karyotype was also processed during each of the FISH rounds, allowing us to monitor the efficiency of probe hybridization.

Analyses of the FISH signals were carried out using direct microscopy by a single observer (Obs. 1) without any knowledge of the fertilization method. Reproducibility was tested by a reanalysis of one image created by the merging of the z-stacked images of the nuclei into one. This reanalysis was performed by Obs. 1 (Obs. 1b) and by a second observer (Obs. 2), both blinded with respect to the fertilization method and previous results.

The following scoring criteria were used to assess the chromosome numbers: In cases where there was doubt if a fluorescence dot was a valid signal or an artifact, the number of signals for that specific chromosome was classified as unknown. Two signals represented two chromosomes if they were a minimum of a signal's width apart.

\section{Data classification}

Embryonic age at fixation was classified as follows: $0-2$ days and 3-5 days, respectively. For each nucleus, the number of signals from one chromosome was classified into three groups: Chromosome count $<3$ (if the number of signals was smaller than 3), chromosome count $=3$ (if the number of signals was equal to 3 ), and chromosome count $>3$ (if the number of signals was larger than 3 ).

\section{Statistical analyses}

A two-tailed Mann-Whitney test was used to test the hypothesis of no difference between categorical baseline data, and a bootstrap likelihood ratio test (10,000 bootstrap samples) was applied for testing the possible differences in the number of nuclei per embryo analyzed between ICSI and IVF embryos [21]. Reproducibility (Obs. 1 vs. Obs. 1b, and Obs. 1 vs. Obs. 2) was tested using the non-parametric Spearman's correlation to compute the correlation coefficient $(r)$ between the counts for each chromosome reanalyzed.

The probabilities of occurrence of chromosome count $<3$ and chromosome count $>3$ were studied using a multivariate Bernoulli (i.e., binomial with size 1) logistic mixed model [22] with three Gaussian random components designed to account for the structural correlation of the experiment. The models included fixed effects representing the fertilization method, the embryonic age, and the chromosome observed. The three nested random components used were as follows: (1) Identity, taking the same value for all the observations arising from the same embryo; (2) Nucleus, taking the same value for each observation arising from the same embryo and the same observed nucleus, and (3) Chromosome identity, taking the same value for each observation from the same embryo, the same observed nucleus, and the same chromosome. In these analyses, the numbers of signals from chromosomes $X$ and $Y$ were combined. The model allowed the use of the three variance components referred above to be different for the observation submitted to different fertilization methods. Note that, due to the basic properties of the Bernoulli logistic model used and because the random components above are nested (in the order given), the probability of two observations arising from the same nucleus and the same individual but related to different chromosomes present the same number of signals increases linearly with the total variance, i.e., the sum of the variances of the three random components $[21,23,24]$. This fact will be explored to characterize the degree of coordination of the number of signals for different chromosomes (i.e., the closeness to euploidy).

The statistical significance of the fixed effects (fertilization method, embryonic age, and chromosome-count) were tested using a parametric bootstrap version of the likelihood ratio test with 10,000 bootstrap samples (here we used a bootstrap version of the likelihood ratio test because the asymptotic distribution of the statistic of the likelihood ratio test under binomial logistic mixed models is not known).

Analyses were performed with Graph Pad version 5.01, R [25] and DMU [26]. Two-sided $p$-values smaller than 0.05 were considered significant

\section{Results}

Sixty-two 3PN embryos were fixated (51 IVF and 11 ICSI). FISH failed in 16 3PN embryos (14 IVF and 2 ICSI). 3PN embryos where only three or fewer FISH probes could be analyzed in all nuclei were excluded from the analysis. In a total of 38 (31 IVF and 7 ICSI) 3PN embryos corresponding to 163 (131 IVF and 32 ICSI) nuclei, the number of signals for 8 different chromosomes were estimated. Baseline data for the embryos analyzed are presented in Table 1. The number of signals was obtained for 993 chromosomes (773 IVF and 220 ICSI), and representative data of the FISH results are shown in Figure 1.

There was a high correlation between the initial chromosome count and the chromosome count made by Obs. $1 \mathrm{~b}$ and Obs. 2 (Table 2). The initial chromosome counts made by Obs. 1 were used for 
further analyses.

Chromosome count $<3$ was found for $69.6 \%$ (691/993) of the chromosomes, chromosome count $=3$ was found for $22.7 \%$ (225/993) of the chromosomes, and chromosome count $>3$ was found for $7.8 \%$ (77/993) of the chromosomes.

The probability of occurrence of chromosome count $<3$ and the probability of occurrence of chromosome count $>3$ were found not to depend on embryonic age nor on the specific chromosome analyzed ( $p=0.21$ and $p=0.31$, respectively). On the other hand, the

Table 1. Data baseline

\begin{tabular}{lccc}
\hline Characteristic & IVF & ICSI & $p$-value \\
\hline No. of women & 28 & 7 & \\
Age of women (yr) & $36 \pm 4.3$ & $29 \pm 5.2$ & $0.0061^{\text {a) }}$ \\
Age of male partner in years & $36 \pm 5.3^{\text {b) }}$ & $32 \pm 4.5$ & $0.083^{c}$ \\
No.of embryos analyzed & 31 & 7 & - \\
No. of nuclei analyzed & 131 & 32 & - \\
No. of embryos reanalyzed (Obs. 1)c & 26 & 6 & - \\
No. of nuclei reanalyzed (Obs. 1)c & 87 & 23 & - \\
No. of embryos analyzed (Obs. 2) & 5 & 4 & - \\
No. of nuclei analyzed (Obs. 2) & 28 & 22 & - \\
Embryonic age (day) & $2.1 \pm 0.97^{\mathrm{c})}$ & $2.4 \pm 0.79$ & $0.34^{\mathrm{a})}$ \\
No. of cells per embryo before fixation & $3.7 \pm 2.5^{\mathrm{d})}$ & $5.3 \pm 3.5$ & - \\
No. of nuclei per embryo after fixation & $3.5 \pm 2.5^{\mathrm{d})}$ & $3.7 \pm 2.4$ & - \\
No. of nuclei analyzed per embryo & $4.2 \pm 2.7$ & $4.6 \pm 3.9$ & $0.95^{\mathrm{e})}$ \\
\hline
\end{tabular}

Values are presented as number or mean \pm standard deviation.

IVF, in vitro fertilization; ICSI, intracytoplasmic sperm-injected; Obs., observer.

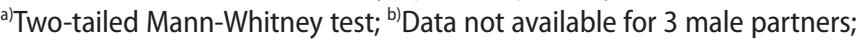
'The embryos were randomly selected within both the IVF and ICSI group;

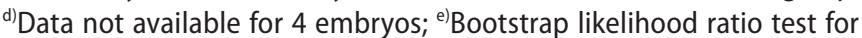
Poisson models for counts. probability of chromosome count $<3$ and chromosome count $>3$ both depended significantly on the fertilization method (IVF or ICSI). Indeed, the difference between the probability of occurrence of chromosome count $<3$ and the probability of occurrence of chromosome count $=3$ were significantly greater for nuclei from 3PN ICSI than for nuclei from 3PN IVF embryos (odds ratio [OR], 5.28; 95\% confidence interval $[\mathrm{Cl}], 1.13-24.75, p<0.001)$ Likewise, the difference between the probability of occurrence of chromosome count $>3$ and the probability of occurrence of chromosome count $=3$ were significantly greater for nuclei from 3PN ICSI than for nuclei from 3PN IVF embryos (OR, 4.35; 95\% Cl, 1.09-17.38; $p<0.001)$.

The probabilities of two observations arising from the same nucleus and the same individual but related to different chromosomes presenting the same number of signals were significantly higher in 3PN ICSI than 3PN IVF fertilized embryos (indeed, the total variances, which increase linearly with the total variance, were $14.47 \pm 3.81$ and $12.87 \pm 1.69$ for ICSI and IVF, respectively, $p<0.001$ ) for chromosomes with chromosome count $<3$. Similar results were found for chromosomes with chromosome count $>3$ (the total variances were $4.123 \pm 2.030$ and $2.87 \pm 1.69$ for ICSI and IVF, respectively, $p=0.002$ ). This indicated a higher degree of coordination of the number of signals for the various chromosomes analyzed in 3PN ICSI embryos than 3PN IVF embryos.

\section{Discussion}

In our cohort, only the fertilization method correlated to differences in chromosome count. In both 3PN IVF and 3PN ICSI embryos, there was a tendency for a reduction in the number of chromosomes from
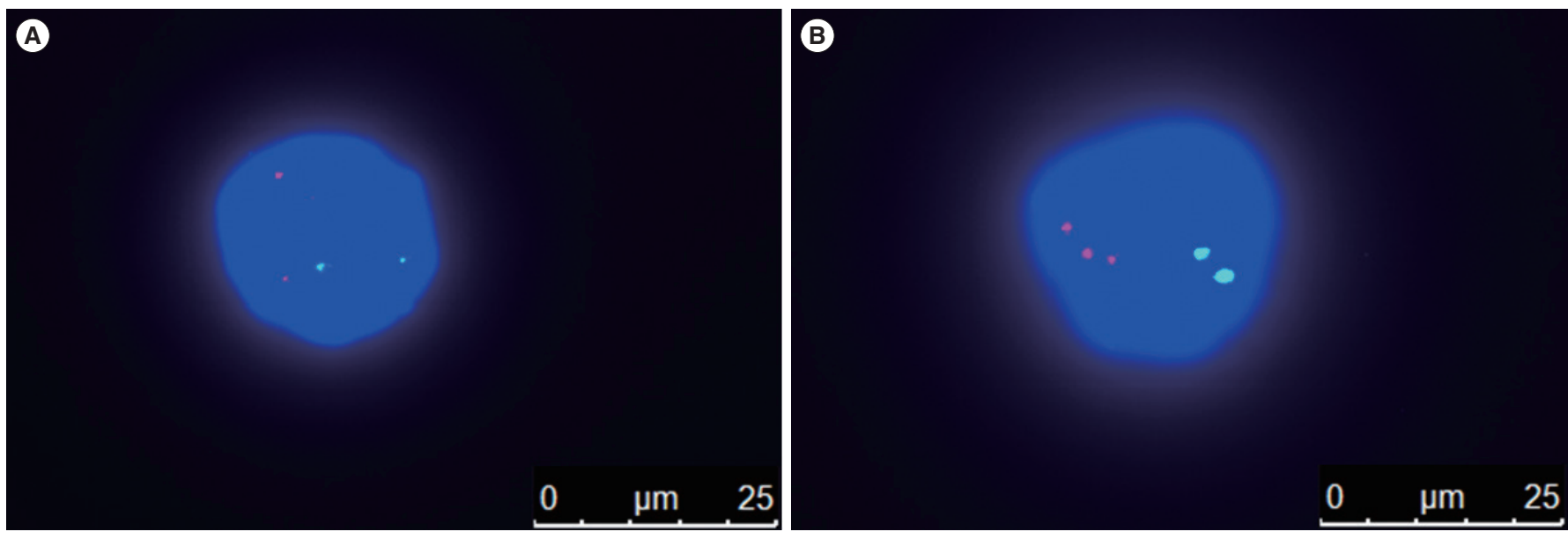

Figure 1. Representative fluorescence in situ hybridization results of intracytoplasmic sperm-injected (ICSI) and in vitro fertilization (IVF) embryos. (A) A nucleus from a three pronuclei (3PN) ICSI embryo containing 2 signals for chromosome 6 (red) and 2 signals for chromosome 11 (green). (B) A nucleus from a 3PN IVF embryo containing 3 signals for chromosome 6 (red) and 2 signals for chromosome 11 (green). The nuclei have been stained with DAPI and a scale bar has been added to the lower right corner of each picture. 
Table 2. Correlations of chromosome counts between observers

\begin{tabular}{|c|c|c|c|c|c|c|c|c|}
\hline \multirow{3}{*}{ Chromosome } & \multicolumn{4}{|c|}{ IVF embryos } & \multicolumn{4}{|c|}{ ICSI embryos } \\
\hline & \multicolumn{2}{|c|}{ Obs. 1 vs. Obs. $1 \mathrm{~b}$} & \multicolumn{2}{|c|}{ Obs. 1 vs. Obs. 2} & \multicolumn{2}{|c|}{ Obs. 1 vs. Obs. $1 \mathrm{~b}$} & \multicolumn{2}{|c|}{ Obs. 1 vs. Obs. 2} \\
\hline & $r$ & $p$-value & $r$ & $p$-value & $r$ & $p$-value & $r$ & $p$-value \\
\hline 6 & 0.81 & $<0.01$ & 0.71 & $<0.01$ & 0.90 & $<0.01$ & 0.82 & $<0.01$ \\
\hline 11 & 0.69 & $<0.01$ & 0.59 & $<0.01$ & 0.83 & $<0.01$ & 0.42 & 0.06 \\
\hline 8 & 0.42 & $<0.01$ & 0.73 & $<0.01$ & 0.82 & $<0.01$ & 0.68 & $<0.01$ \\
\hline 17 & 0.53 & $<0.01$ & 0.43 & 0.03 & 0.76 & $<0.01$ & 0.71 & $<0.01$ \\
\hline 10 & 0.49 & $<0.01$ & 0.30 & 0.16 & 0.64 & $<0.01$ & 0.83 & $<0.01$ \\
\hline 18 & 0.66 & $<0.01$ & 0.71 & $<0.01$ & 0.86 & $<0.01$ & 0.95 & $<0.01$ \\
\hline$x$ & 0.45 & $<0.01$ & 0.73 & $<0.01$ & 0.88 & $<0.01$ & 0.66 & $<0.01$ \\
\hline $\mathrm{Y}$ & 0.37 & 0.01 & 0.84 & $<0.01$ & 0.75 & $<0.01$ & -0.15 & 0.58 \\
\hline
\end{tabular}

IVF, in vitro fertilization; ICSI, intra cytoplasmic sperm injection; Obs. 1, chromosome counts by first observer; Obs. 1b, reanalysis and new chromosome counts by observer 1 blinded for initial chromosome counts; Obs. 2, chromosome counts by the second observer; $r$, spearman correlation coefficient; $p$-value, the 2 -sided $p$-values reflect whether there was no correlation between chromosome counts (null hypothesis) against the alternative that there was a correlation (alternative hypothesis).

the assumed number of 3 copies of each chromosome in the 3PN zygote. In 3PN ICSI embryos, the probability of a chromosome count below or above 3 was significantly higher than in 3PN IVF embryos. A higher degree of coordination of the number of signals for different chromosomes were found in 3PN ICSI embryos compared to 3PN IVF embryos, indicating that the karyotypes of 3PN ICSI embryos were closer to euploid than those of 3PN IVF embryos.

\section{Limitations to the study}

FISH analysis for a limited number of chromosomes was used to estimate the chromosomal status of the 3PN embryos. Therefore, we were not able evaluate untested chromosomes. Likewise, FISH does not allow for detection of partial deletions or duplications of chromosomes since the probe hybridizes to a specific locus or the centromere, and hence only provides information about that segment of the chromosome [27]. Another technical limitation of FISH is that errors in chromosome count can occur due to loss or damage of nuclear material; split, diffused, or overlapping signals; hybridization failure; or probe inefficiency. As FISH analyses have approximately $95 \%$ accuracy per probe, using a multiprobe panel on one nucleus from a 3PN embryo, potentially with several signals per probe, renders the risk of misdiagnosis significant [28,29]. Nevertheless, FISH is as reliable technique for ploidy determination for research purposes [29]. Moreover, we controlled the chromosome count by evaluating the reproducibility and found high intra- and inter-observer correlations. FISH failed in some embryos and as only high quality FISH results were analyzed, a smaller cohort was available for further analyses. Despite the relatively small cohort analyzed, significant differences were found. Our data does not, however, allow for exclusion of variables other than the fertilization method (for example maternal/paternal age), even though they also could impact the karyotype of the embryos.

\section{Chromosomal makeup of 3PN embryos}

Although it has been demonstrated that 3PN zygotes generally have 3 haploid sets of chromosomes, several studies of 3PN embryos have revealed triploid, diploid, or severely abnormal karyotypes $[3,4,6,8,10,12-19,30]$. However, studies reporting mosaic chromosome imbalances in normal 2PN in vitro fertilized embryos showed that chromosome instability also occurs during normal human embryogenesis [31-40]. This instability could be a result of the inactive embryonic genome rendering normal mechanisms for induction of apoptosis in chromosomal abnormal cells impossible [33,34,38]. This is supported by reports suggesting a decrease in the proportion of aneuploid cells at the blastocyst stage $[33,35,38]$.

The aneuploidy and mosaicism previously reported in 3PN embryos $[3,4,6,8,10,12-19,30]$ could, therefore, be a "normal" developmental event with an etiology as mentioned above and not a consequence of the 3PN status.

If the aneuploidy and mosaicism found in 3PN embryos were the effect of a "normal" developmental event alone, there would presumably be no difference in the degree of coordination of the number of signals for different chromosomes in 3PN ICSI and IVF embryos.

However, 3PN ICSI nuclei displayed a higher degree of coordination between numbers of signals for different chromosomes than 3PN IVF nuclei. A possible explanation might be different numbers of centrioles in the 3PN zygote.

In the human zygote the first mitotic division is controlled by the paternally inherited centriole $[10,11]$. Fertilization with two spermatozoa introduces 2 centrioles into the oocyte. Abnormal cleavage directly into 3 cells, has been reported in cells with extra centrioles $[5,7,10,11,41]$. The vast majority of 3PN IVF zygotes are of dispermic 
origin and thus more likely to have extra centrioles [42]. Concordantly, we have previously observed that significantly more 3PN IVF zygotes cleaved early into 3 cells-defined as a duration of the 2-cell stage of less than 1 hour-than either 3PN ICSI or 2PN IVF zygotes [43]. If, in fact, the cells showing "early cleavage into 3 cells" actually undergo direct cleavage into 3 cells, then the 3PN IVF embryos would be expected to display a reduction in the number of chromosomes per cell, since the triploid genome - after duplication and syngamy of the pronuclei-is divided into 3 cells instead of the normal 2. This could explain why 3PN IVF nuclei in this study tended to have a chromosome count $<3$.

The different cleavage patterns observed for 3PN IVF and 3PN ICSI embryos do, however, not explain why 3PN ICSI nuclei were more likely than the 3PN IVF nuclei to display a chromosome count below or above 3. The 3PN ICSI nuclei displayed a higher coordination of the number of signals for different chromosomes than the 3PN IVF nuclei, which could indicate that 3PN ICSI nuclei possess a mechanism of euploid ploidy reduction (removal of haploid set(s) of chromosomes). As Grau et al. [19] have found that self-correction in 3PN ICSI embryos apparently takes place before day 2 of embryo development and hence, before embryonic genome activation, we speculate that the self-correction is independent of genomic content but caused by an insufficient number of centrioles relative to the number of pronuclei. Such an insufficiency could hamper the pronuclear apposition of all pronuclei after fertilization.

No studies have analyzed the pronuclear apposition in detail in humans. However, studies of other species have shown that pronuclear apposition is dependent on the ability of the sperm asters to both push the male pronucleus further into the cytoplasm, and to reach the female pronucleus allowing it to be moved toward the male pronucleus [44,45].

We, therefore, speculate that this apparent ability to self-correct or maintain polyploidy in 3PN ICSI embryos, is caused by an insufficient number of sperm asters relative to the number of pronuclei, speculating that one sperm aster may not always form adequate numbers of microtubules to allow 3 pronuclei to assemble. In that case, this self-correction of the polyploidy would be less likely in the 3PN IVF embryos.

Our results show that the fertilization method (IVF or ICSI) impacts the karyotype of the 3PN embryos. The chromosome count in the ICSI nuclei tended to differ more from 3 and were closer to euploidy than that in the 3PN IVF nuclei. This could indicate that the number of centrioles in the 3PN zygote influences the karyotype.

\section{Conflict of interest}

No potential conflict of interest relevant to this article was reported.

\section{Acknowledgments}

The authors wish to thank the clinical, the paramedical, and the laboratory team of the Fertility Clinic, Braedstrup/Horsens Hospital.

\section{References}

1. Jacobs PA, Szulman AE, Funkhouser J, Matsuura JS, Wilson CC. Human triploidy: relationship between parental origin of the additional haploid complement and development of partial hydatidiform mole. Ann Hum Genet 1982;46:223-31.

2. Jauniaux E, Brown R, Rodeck C, Nicolaides KH. Prenatal diagnosis of triploidy during the second trimester of pregnancy. Obstet Gynecol 1996;88:983-9.

3. Grossmann M, Calafell JM, Brandy N, Vanrell JA, Rubio C, Pellicer A, et al. Origin of tripronucleate zygotes after intracytoplasmic sperm injection. Hum Reprod 1997;12:2762-5.

4. Staessen C, Van Steirteghem AC. The chromosomal constitution of embryos developing from abnormally fertilized oocytes after intracytoplasmic sperm injection and conventional in-vitro fertilization. Hum Reprod 1997;12:321-7.

5. Yu SL, Lee RK, Su JT, Chih YF, Tsai YC, Lin MH, et al. Distinction between paternal and maternal contributions to the tripronucleus in human zygotes obtained after in vitro fertilization. Taiwan J Obstet Gynecol 2006;45:313-6.

6. Macas E, Imthurn B, Rosselli M, Keller PJ. The chromosomal complements of multipronuclear human zygotes resulting from intracytoplasmic sperm injection. Hum Reprod 1996;11:2496-501.

7. Plachot $\mathrm{M}$, Crozet N. Fertilization abnormalities in human in-vitro fertilization. Hum Reprod 1992;7 Suppl 1:89-94.

8. Angell RR, Templeton AA, Messinis IE. Consequences of polyspermy in man. Cytogenet Cell Genet 1986;42:1-7.

9. Plachot M, Mandelbaum J, Junca AM, de Grouchy J, Salat-Baroux J, Cohen J. Cytogenetic analysis and developmental capacity of normal and abnormal embryos after IVF. Hum Reprod 1989;4:99103.

10. Palermo G, Munne $\mathrm{S}$, Cohen J. The human zygote inherits its mitotic potential from the male gamete. Hum Reprod 1994;9:1220-5.

11. Sathananthan AH, Kola I, Osborne J, Trounson A, Ng SC, Bongso A, et al. Centrioles in the beginning of human development. Proc Natl Acad Sci U S A 1991;88:4806-10.

12. Chen $X$, Luo Y, Fan Y, Yue L, Wu X, Chen Y, et al. Triploid and diploid embryonic stem cell lines derived from tripronuclear human zygotes. J Assist Reprod Genet 2012;29:713-21.

13. Kola I, Trounson A, Dawson G, Rogers P. Tripronuclear human oocytes: altered cleavage patterns and subsequent karyotypic analysis of embryos. Biol Reprod 1987;37:395-401. 
14. Pieters MH, Dumoulin JC, Ignoul-Vanvuchelen RC, Bras M, Evers $J \mathrm{~L}$, Geraedts JP. Triploidy after in vitro fertilization: cytogenetic analysis of human zygotes and embryos. J Assist Reprod Genet 1992;9:68-76.

15. Rosenbusch B, Schneider M, Sterzik K. The chromosomal constitution of multipronuclear zygotes resulting from in-vitro fertilization. Hum Reprod 1997;12:2257-62.

16. Coonen E, Harper JC, Ramaekers FC, Delhanty JD, Hopman AH, Geraedts JP, et al. Presence of chromosomal mosaicism in abnormal preimplantation embryos detected by fluorescence in situ hybridisation. Hum Genet 1994;94:609-15.

17. Pang MG, Jee BC, Kim SH, Ryu BY, Oh SK, Suh CS, et al. Chromosomal constitution of embryos derived from tripronuclear zygotes studied by fluorescence in situ hybridization using probes for chromosomes 4, 13, 18, 21, X, and Y. Gynecol Obstet Invest 2005;59:14-8.

18. Lim AS, Goh VH, Su CL, Yu SL. Microscopic assessment of pronuclear embryos is not definitive. Hum Genet 2000;107:62-8.

19. Grau N, Escrich L, Martin J, Rubio C, Pellicer A, Escriba MJ. Selfcorrection in tripronucleated human embryos. Fertil Steril 2011; 96:951-6.

20. Mantikou E, van Echten-Arends J, Sikkema-Raddatz B, van der Veen F, Repping S, Mastenbroek S. Temporal and developmentalstage variation in the occurrence of mitotic errors in tripronuclear human preimplantation embryos. Biol Reprod 2013;89:42.

21. Joergensen B, Labouriau R. Exponential families and theoretical inference. 2nd ed. New York: Springer; 2012.

22. Maia RP, Ask B, Madsen P, Pedersen J, Labouriau R. Genetic determination of mortality rate in Danish dairy cows: a multivariate competing risk analysis based on the number of survived lactations. J Dairy Sci 2014;97:1753-61.

23. Joergensen B, Labouriau R, Lunbdye-Christensen S. Linear Growth curve analysis based on exponential dispersion models. J R Stat Soc Ser B 1996;58:573-92.

24. Maia RP, Madsen P, Labouriau R. Multivariate Survival Mixed Models for Genetic Analysis of Longevity Traits. Journal of Applied Statistics 2014;41:1286-1306.

25. R Core Team. R: A language and environment for statistical computing [Internet]. Vienna, Austria; R Foundation for Statistical Computing; 2013 [cited 2014 Feb 25]. Available from: http:// www.R-project.org/.

26. Madsen P, Su G, Labouriau R, Christensen OF. DMU-a package for analyzing multivariate mixed models. Hannover: Gesellschaft für Tierzuchtwissenschaft e. V.; 2010.

27. Wilton L, Voullaire L, Sargeant P, Williamson R, McBain J. Preimplantation aneuploidy screening using comparative genomic hybridization or fluorescence in situ hybridization of embryos from patients with recurrent implantation failure. Fertil Steril 2003; 80:860-8.

28. Ruangvutilert P, Delhanty JD, Rodeck CH, Harper JC. Relative efficiency of FISH on metaphase and interphase nuclei from nonmosaic trisomic or triploid fibroblast cultures. Prenat Diagn 2000; 20:159-62.

29. DeUgarte CM, Li M, Surrey M, Danzer H, Hill D, DeCherney AH. Accuracy of FISH analysis in predicting chromosomal status in patients undergoing preimplantation genetic diagnosis. Fertil Steril 2008;90:1049-54.

30. Macas E, Imthurn B, Roselli M, Keller PJ. Chromosome analysis of single- and multipronucleated human zygotes proceeded after the intracytoplasmic sperm injection procedure. J Assist Reprod Genet 1996;13:345-50.

31. Delhanty JD, Harper JC, Ao A, Handyside AH, Winston RM. Multicolour FISH detects frequent chromosomal mosaicism and chaotic division in normal preimplantation embryos from fertile patients. Hum Genet 1997;99:755-60.

32. Voullaire L, Slater H, Williamson R, Wilton L. Chromosome analysis of blastomeres from human embryos by using comparative genomic hybridization. Hum Genet 2000;106:210-7.

33. Fragouli E, Lenzi M, Ross R, Katz-Jaffe M, Schoolcraft WB, Wells D. Comprehensive molecular cytogenetic analysis of the human blastocyst stage. Hum Reprod 2008;23:2596-608.

34. Vanneste E, Voet T, Le Caignec C, Ampe M, Konings P, Melotte C, et al. Chromosome instability is common in human cleavagestage embryos. Nat Med 2009;15:577-83.

35. Fragouli E, Alfarawati S, Daphnis DD, Goodall NN, Mania A, Griffiths T, et al. Cytogenetic analysis of human blastocysts with the use of FISH, CGH and aCGH: scientific data and technical evaluation. Hum Reprod 2011;26:480-90.

36. Mertzanidou A, Wilton L, Cheng J, Spits C, Vanneste E, Moreau Y, et al. Microarray analysis reveals abnormal chromosomal complements in over $70 \%$ of 14 normally developing human embryos. Hum Reprod 2013;28:256-64.

37. Mertzanidou A, Spits C, Nguyen HT, Van de Velde H, Sermon K. Evolution of aneuploidy up to Day 4 of human preimplantation development. Hum Reprod 2013;28:1716-24.

38. Barbash-Hazan S, Frumkin T, Malcov M, Yaron Y, Cohen T, Azem F, et al. Preimplantation aneuploid embryos undergo self-correction in correlation with their developmental potential. Fertil Steril 2009;92:890-6.

39. Yang Z, Zhang J, Salem SA, Liu X, Kuang Y, Salem RD, et al. Selection of competent blastocysts for transfer by combining timelapse monitoring and array CGH testing for patients undergoing preimplantation genetic screening: a prospective study with sibling oocytes. BMC Med Genomics 2014;7:38. 
40. Liu J, Sills ES, Yang Z, Salem SA, Rahil T, Collins GS, et al. Array comparative genomic hybridization screening in IVF significantly reduces number of embryos available for cryopreservation. Clin Exp Reprod Med 2012;39:52-7.

41. Sathananthan AH, Ratnam SS, Ng SC, Tarin JJ, Gianaroli L, Trounson $A$. The sperm centriole: its inheritance, replication and perpetuation in early human embryos. Hum Reprod 1996;11:345-56.

42. Simerly C, Wu GJ, Zoran S, Ord T, Rawlins R, Jones J, et al. The paternal inheritance of the centrosome, the cell's microtubule-organizing center, in humans, and the implications for infertility. Nat Med 1995;1:47-52.
43. Joergensen MW, Agerholm I, Hindkjaer J, Bolund L, Sunde L, Ingerslev $\mathrm{HJ}$, et al. Altered cleavage patterns in human tripronuclear embryos and their association to fertilization method: a timelapse study. J Assist Reprod Genet 2014;31:435-42.

44. Reinsch S, Gonczy P. Mechanisms of nuclear positioning. J Cell Sci 1998;111 ( Pt 16):2283-95.

45. Gonczy P, Schnabel H, Kaletta T, Amores AD, Hyman T, Schnabel R. Dissection of cell division processes in the one cell stage Caenorhabditis elegans embryo by mutational analysis. J Cell Biol 1999;144:927-46. 